\title{
Efeitos da Administração Subaracnóidea de Grandes Volumes de Lidocaína a $2 \%$ e Ropivacaína a $1 \%$ sobre a Medula Espinhal e as Meninges. Estudo Experimental em Cães *
}

\section{Effects of Spinal Administration of Large Volumes of 2\% Lidocaine and $1 \%$ Ropivacaine on Spinal Cord and Meninges. Experimental Study in Dogs}

Eliana Marisa Ganem TSA ${ }^{1}$, Pedro Thadeu Galvão Vianna TSA ${ }^{2}$, Mariângela Marques ${ }^{3}$, Yara Marcondes Machado Castiglia, TSA ${ }^{2}$, Luiz Antonio Vane, TSA ${ }^{2}$

\begin{abstract}
RESUMO
Ganem EM, Vianna PTG, Marques M, Castiglia YMM, Vane LA Efeitos da Administração Subaracnóidea de Grandes Volumes de Lidocaína a $2 \%$ e Ropivacaína a 1\% sobre a Medula Espinhal e as Meninges. Estudo Experimental em Cães
\end{abstract}

JUSTIFICATIVA E OBJETIVOS: A injeção de grandes volumes de anestésico local no espaço subaracnóideo, após punção dural acidental, é complicação da anestesia peridural. O objetivo desta pesquisa foi investigar as possíveis alterações clínicas e histológicas desencadeadas por grandes volumes de lidocaína a $2 \%$ e ropivacaína a $1 \%$, simulando injeção subaracnóidea acidental, em cães.

MÉTODO: Vinte e um cães foram distribuídos aleatoriamente em 3 grupos, que receberam por via subaracnóidea: G1 cloreto de sódio a 0,9\%; G2 - lidocaína a 2\% e G3 - ropivacaína a $1 \%$. A punção subaracnóidea foi realizada no espaço intervertebral $L_{6}-L_{7}$. O volume de anestésico local administrado foi de $1 \mathrm{ml}$ para cada $10 \mathrm{~cm}$ de distância entre a protuberância occipital e o espaço lombossacral (5 - 6,6 ml). Após 72 horas de observação clínica os animais foram sacrificados e foi removida a porção lombossacral da medula para exame histológico, por microscopia óptica.

RESULTADOS: Nenhum animal do G1 apresentou alterações clínicas ou histológicas da medula espinhal. Foram observados dois casos de necrose do tecido nervoso em G2, porém mudanças clínicas, em somente um desses cães e em outros dois animais que não apresentaram alterações histológicas. Foi encontrada necrose focal do tecido nervoso medular em um animal de G3. Todos os animais de G3 permaneceram clinicamente normais.

* Recebido do (Received from) CET/SBA do Departamento de Anestesiologia da Faculdade de Medicina de Botucatu (FMB-UNESP), Botucatu, $S P$

1. Professora Adjunta Livre Docente do CET/SBA do Departamento de Anestesiologia da FMB-UNESP

2. Prof. (a) Titular do CET/SBA do Departamento de Anestesiologia da FMB-UNESP

3. Prof ${ }^{a}$ Assistente Doutora do Departamento de Patologia da FMB-UNESP

Endereço para correspondência (Correspondence to)

Profa. Dra. Eliana Marisa Ganem

Departamento de Anestesiologia da FMB-UNESP

Distrito de Rubião Júnior

18618-970 - Botucatu, SP

E-mail:eganem@fmb.unesp.br

Apresentado (Submitted) em 23 de setembro de 2002

Aceito (Accepted) para publicação 22 de novembro de 2002

(C) Sociedade Brasileira de Anestesiologia, 2003

Revista Brasileira de Anestesiologia

Vol. 53, № 3, Maio - Junho, 2003
CONCLUSÕES: Conclui-se que grandes volumes de lidocaína a $2 \%$ determinaram alterações clínicas e histológicas mais intensas que os de ropivacaína a $1 \%$.

Unitermos: ANESTÉSICOS, Local: lidocaína, ropivacaína; ANIMAL: Cão; COMPLICAÇÕES: lesão neurológica, neutoxicidade; TÉCNICAS ANESTÉSICAS, Regional: subaracnóidea

\section{SUMMARY}

Ganem EM, Vianna PTG, Marques M, Castiglia YMM, Vane LA Effects of Spinal Administration of Large Volumes of $2 \%$ Lidocaine and 1\% Ropivacaine on Spinal Cord and Meninges. Experimental Study in Dogs

BACKGROUND AND OBJECTIVES: Spinal injection of large local anesthetic volumes after accidental dural puncture is an epidural anesthesia complication. This study aimed at investigating potential clinical and histological changes triggered by large volumes of $2 \%$ lidocaine or $1 \%$ ropivacaine in a simulated accidental spinal injection in dogs.

METHODS: Twenty one dogs were randomly allocated into three experimental groups, which received spinal injections of: G1 - $0.9 \%$ sodium chloride, G2 - 2\% lidocaine, G3 - 1\% ropivacaine. Spinal puncture was performed in $L_{6}-L_{7}$ interspace. Anesthetic volume was $1 \mathrm{ml}$ per $10 \mathrm{~cm}$-distance between the occipital protuberance and the lumbosacral space (5 - $6.6 \mathrm{ml}$ ). After 72 hours of clinical observation animals were sacrificed and their spinal cords were removed for histological examination under light microscopy.

RESULTS: No G1 animal presented clinical or histological changes in the spinal cord. There were two cases of nervous tissue necrosis in G2, however clinical changes were only observed in one of these dogs and in two other dogs which had no histological changes. There has been focal necrosis in the spinal cord nervous tissue of one G3 animal. All G3 animals remained clinically normal.

CONCLUSIONS: Large volumes of $2 \%$ lidocaine have determined more intensive clinical and histological changes as compared to $1 \%$ ropivacaine.

Key Words: ANESTHETICS, Local: lidocaine, ropivacaine; ANESTHETIC TECHNIQUES, Regional: spinal block; ANIMAL: Dog; COMPLICATIONS: neurologic damage, neurotoxicity

\section{INTRODUÇÃO}

s anestésicos locais são fármacos que bloqueiam, de forma reversível, os canais de sódio da membrana celular, impedindo, dessa forma, a condução dos impulsos dos tecidos excitáveis ${ }^{1}$. 
A lidocaína, anestésico do grupo aminoamida, é muito utilizada em decorrência de seu rápido início de ação, sua potência e seu moderado tempo de ação ${ }^{2}$. Sintetizada em 1948 por Löfgren, foi utilizada pela primeira vez pela via subaracnóidea na concentração de $2 \%{ }^{3}$. Na concentração de $5 \%$, foi introduzida em anestesia em meados de 1950. A partir de então, passou a ser empregada sem que se tivesse realizado pesquisa que avaliasse os riscos de possíveis lesões neurológicas desencadeadas pela sua administração no espaço subaracnóideo. Em 1969, foi publicado resultado de estudo multicêntrico, envolvendo 10.440 pacientes, a maioria da clínica obstétrica, nas quais não foi observada nenhuma lesão neurológica grave ${ }^{4}$.

Entretanto, na década de 1990 foram descritos vários casos de síndrome de cauda eqüina, que foram associados à lidocaína hiperbárica a 5\% administrada no espaço subaracnóideo através de microcateter ${ }^{5,6}$, de agulhas de ponta de lápis de pequeno calibre ${ }^{7}$, após injeções repetidas em decorrência de bloqueios inadequados ${ }^{7,8} \mathrm{e}$, finalmente, após a injeção de grandes volumes de lidocaína a $2 \%$ que deveria, inicialmente, ser administrada no espaço peridural e que acidentalmente foi introduzida no espaço subaracnóideo ${ }^{\text {9-11. }}$.

Asíndrome da cauda eqüina consiste de disfunção urinária e intestinal, perda de sensibilidade na região períneal e graus variáveis de fraqueza muscular nos membros inferiores.

Quanto à ropivacaína, anestésico do grupo aminoamida, preparado como enantiômero S puro, nada se sabe sobre sua capacidade de desencadear neurotoxicidade ${ }^{12}$.

O objetivo desta pesquisa foi avaliar os efeitos que grandes volumes de lidocaína a $2 \%$ e ropivacaína a $1 \%$, administrados por via subaracnóidea, determinariam sobre a histologia do tecido nervoso da medula espinhal e das meninges de cães.

\section{MÉTODO}

Após a aprovação pelo Comitê de Ética em Experimentação Animal, foram utilizados 21 cães adultos, de ambos os sexos, sem raça definida, cujos pesos variaram de 5 a $14 \mathrm{~kg}$ e comprimentos de coluna vertebral de 50 a $66 \mathrm{~cm}$. Os cães foram distribuídos aleatoriamente em 3 grupos de 7 animais, que se diferenciaram pela solução administrada no espaço subaracnóideo, ou seja: grupo 1 - solução fisiológica, grupo 2 - lidocaína a 2\%, grupo 3 - ropivacaína a 1\%.

Em todos os animais foi realizada a mesma seqüência experimental. Após jejum de 12 horas com livre acesso a água, os cães receberam $30 \mathrm{mg} \cdot \mathrm{kg}^{-1}$ de pentobarbital sódico por via venosa, foram intubados e mantidos em respiração espontânea em circuito circular com fração inspirada de oxigênio a $100 \%$. Foram realizadas dissecções e cateterismos da veia femoral direita para hidratação com solução de Ringer com lactato, e da artéria femoral direita para medida da pressão arterial média.

Foi instalado oxímetro de pulso para medida da saturação periférica de oxigênio, cujo sensor foi colocado na língua do cão, e instalado o conector para medida da fração expirada de dióxido de carbono $\left(\mathrm{CO}_{2}\right)$, junto à cânula de intubação. Os animais foram colocados em decúbito ventral na goteira de Claude Bernard, tendo sido medida a distância entre a protuberância occipital e o espaço lombossacral para obtenção do comprimento da coluna vertebral. Este comprimento foi utilizado para calcular o volume da solução a ser administrado no espaço subaracnóideo.

Foi realizada a limpeza da pele e dos pelos, com água e sabão, em área de aproximadamente $10 \mathrm{~cm}$ ao redor dos espaços $L_{6}-L_{7}$, seguida de lavagem com água, anti-sepsia com álcool iodado e colocação de campos estéreis.

Foi realizada a palpação das duas tuberosidades do osso ilíaco e do processo espinhoso da última vértebra lombar. Imediatamente abaixo, localizava-se o espaço lombossacral. Deslizando-se o indicador em direção cefálica, o próximo espaço intervertebral era o $L_{6}-L_{7}$.

Para realização da punção subaracnóidea, introduzia-se, através de acesso mediano, com ângulo de inclinação de aproximadamente $45^{\circ}$, a agulha de Quincke, calibre 22G, descartável, com o bisel da agulha direcionado cefalicamente. Ao ultrapassar a membrana aracnóide, retirava-se o mandril da agulha e obtinha-se o escoamento do líquor.

Asolução previamente sorteada era injetada lentamente, durante 1 minuto, em volume que correspondia a 1 mililitro para cada 10 centímetros de distância entre a protuberância occipital e o espaço lombo-sacro ${ }^{13}$.

Após a injeção do anestésico local, os animais eram colocados em decúbito dorsal, sendo observadas a freqüência cardíaca, a pressão arterial média e a amplitude e a freqüência dos movimentos respiratórios. Estes parâmetros auxiliaram na avaliação da extensão do bloqueio anestésico.

Os animais que apresentavam hipotensão arterial (pressão arterial média inferior a 30\% dos valores iniciais) recebiam volume adicional de solução de Ringer com lactato (20 $\left.\mathrm{ml} . \mathrm{kg}^{-1}\right)$. Naqueles cuja diminuição da pressão arterial média era maior ou igual a $50 \%$, administrou-se sulfato de efedrina na dose de $0,5 \mathrm{mg} \cdot \mathrm{kg}^{-1}$.

Nos cães que apresentavam bradicardia (freqüência cardíaca $\leq 50 \%$ dos valores obtidos antes da anestesia subaracnóidea ou $\leq 60$ batimentos por minuto) administrava-se sulfato de atropina $20 \mu \mathrm{g} \cdot \mathrm{kg}^{-1}$.

Nos animais que apresentavam parada respiratória, era instituída ventilação mecânica controlada até a recuperação de ventilação espontânea eficaz.

Também foram observados, durante o período em que o animal estava em vigência do bloqueio subaracnóideo, o grau de relaxamento do esfíncter anal e a sensibilidade dolorosa nos diferentes dermátomos, como membrana interdigital traseira e dianteira, cauda e diferentes dermátomos das regiões cervical, torácica, lombar e sacral, com auxílio de pinça com dente de rato.

Após a recuperação da anestesia subaracnóidea e venosa, os animais permaneceram sob observação clínica durante as 72 horas em que foram mantidos no cativeiro. Foram avaliados: relaxamento do esfíncter anal, perda da força muscu- 
lar das patas posteriores, capacidade de movimentar a cauda e alteração da sensibilidade dolorosa.

Os animais foram sacrificados por eletrocussão, após anestesia prévia e foram retiradas as porções lombar e sacral da medula espinhal em tempo inferior a 3 minutos, sendo fixadas em solução de formalina a $10 \%$.

Foram realizados cortes transversais do tecido nervoso e das meninges, que se iniciaram aproximadamente $5 \mathrm{~cm}$ acima do local onde foi realizada a punção subaracnóidea, indo até o final da cauda eqüina, em intervalos de $1 \mathrm{~cm}$.

Os cortes foram corados pelo método de hematoxilina-eosina e a leitura das lâminas foi efetuada pela microscopia óptica, sem que o patologista soubesse a que grupo experimental pertencia a lâmina.

Tendo o objetivo de avaliar a homogeneidade dos grupos com relação ao peso e ao comprimento da coluna vertebral, foi realizada a Análise de Variância, sendo considerados significativos os valores de $p<0,05$.

\section{RESULTADOS}

Não houve diferença significativa entre os grupos com relação ao peso e ao comprimento da coluna vertebral (Tabela I).

Tabela I - Resultados Referentes ao Peso (kg) e ao Comprimento de Coluna Vertebral $(\mathrm{cm})$ dos Animais Pertencentes aos 3 Grupos Experimentais (Média \pm DP)

\begin{tabular}{|c|c|c|c|}
\hline & $\begin{array}{c}\text { Solução } \\
\text { Fisiológica } \\
\text { (G1) }\end{array}$ & $\begin{array}{c}\text { Lidocaína a 2\% } \\
\text { G2) }\end{array}$ & $\begin{array}{c}\text { Ropivacaína a 1\% } \\
\text { (G3) }\end{array}$ \\
\hline Peso (kg) & $9,6 \pm 2,8$ & $9,1 \pm 1,9$ & $9,3 \pm 2,9$ \\
\hline $\begin{array}{l}\text { Comprimento da } \\
\text { coluna vertebral }(\mathrm{cm})\end{array}$ & $59 \pm 6$ & $62 \pm 5$ & $60 \pm 6$ \\
\hline
\end{tabular}

Todos os animais pertencentes aos grupos 2 e 3 necessitaram de ventilação mecânica após a realização do bloqueio subaracnóideo, porque apresentaram paralisia da musculatura respiratória e conseqüente parada respiratória. Também apresentaram hipotensão arterial. Em onze animais, a redução da pressão variou de $20 \%$ a $50 \%$; foi transitória e corrigida com aumento da hidratação por via venosa. Nos 3 restantes houve necessidade de se administrar sulfato de efedrina.

Todos os animais dos grupos 2 e 3 apresentaram diminuição da freqüência cardíaca, que variou de $40 \%$ a $75 \%$ dos valores obtidos antes da anestesia subaracnóidea. Entretanto, apenas quatro cães necessitaram de sulfato de atropina para restabelecimento da freqüência cardíaca.

Nenhum animal do grupo 1 apresentou alterações clínicas ou histológicas da medula espinhal (Figura 1).

Foram observadas alterações histológicas em dois animais pertencentes ao grupo 2 e alterações clínicas em um deles e em outros dois cães deste grupo, os quais, por sua vez, não apresentaram alterações histológicas. Em um dos animais que apresentaram alterações histológicas constatou-se necrose em faixa de toda superfície medular e do fascículo de gracilis, caracterizada por vacuolização com perda de substância em $75 \%$ do campo histológico, $2 \mathrm{~cm}$ acima do local onde foi realizada a punção (Figura 2). Sua avaliação clínica mostrou paralisia das patas posteriores e relaxamento do esfíncter anal. No segundo animal, detectou-se foco de necrose no cone medular, em extensão que ocupou $30 \%$ do campo histológico, não tendo sido observadas alterações clínicas.

Em dois animais, que permaneceram com o esfíncter anal parcialmente relaxado após o bloqueio, não foram encontradas alterações histológicas no tecido nervoso espinhal e nas meninges (Quadro I).

Em um animal do grupo 3 foi observada área focal de vacuolização na superfície dorso-lateral da medula e na raiz dorsal, ocupando $5 \%$ do campo histológico, aproximadamente $2 \mathrm{~cm}$ acima do local onde foi realizada a punção (Figura 3). Todos os animais pertencentes a este grupo permaneceram clinicamente normais (Quadro II).

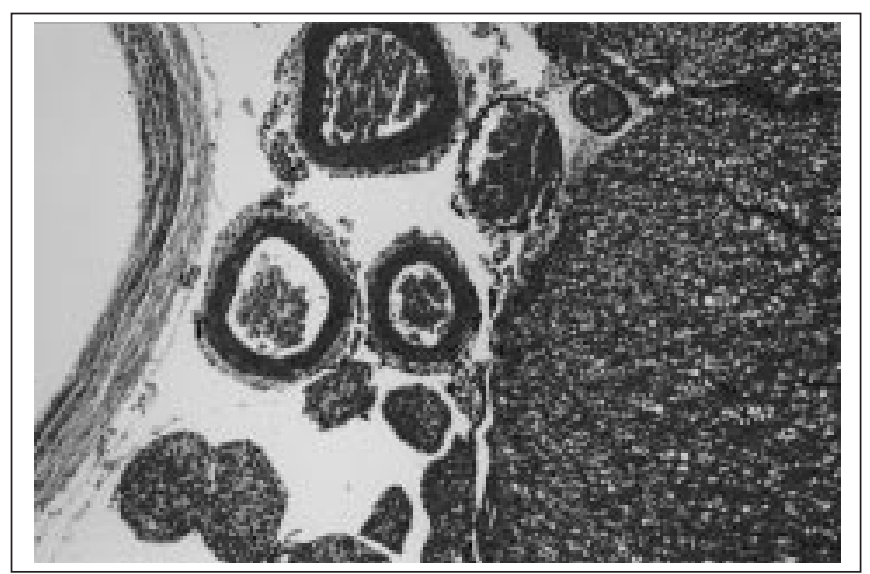

Figura 1 - Tecido Nervoso, Vasos Sangüíneos e Meninges Normais de Animal do Grupo 1. H E 100x

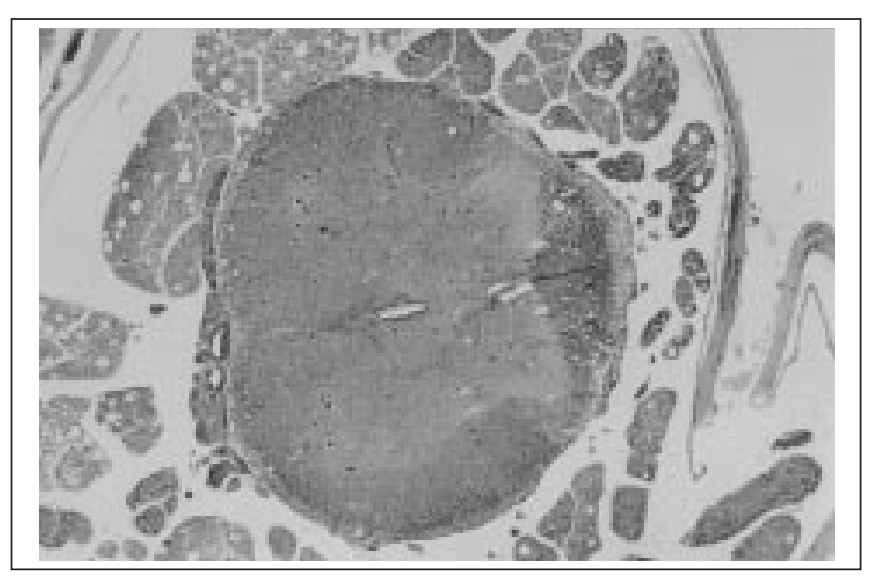

Figura 2 - Necrose em Faixa de toda a Superfície Medular do Fascículo de Gracilis de Animal do Grupo 2. H E 20x 
Quadro I - Observações Clínicas e Histológicas dos 7 Cães Pertencentes ao Grupo 2 que Receberam Lidocaína a $2 \%$

\begin{tabular}{lll}
\hline Cão & Observações clínicas & Observações histológicas \\
\hline 1 & $\begin{array}{l}\text { Paralisia das patas posteriores e } \\
\text { relaxamento total do esfíncter anal }\end{array}$ & $\begin{array}{l}\text { Necrose em faixa atingindo } \\
\text { toda a superfície medular e o } \\
\text { fascículo de gracilis (75\%) }\end{array}$ \\
2 & Relaxamento parcial do esfíncter anal & Normal \\
3 & Relaxamento parcial do esfíncter anal & Normal \\
4 & Normal & Normal \\
5 & Normal & $\begin{array}{l}\text { Foco de necrose no cone } \\
\text { medular (30\%) }\end{array}$ \\
6 & Normal & Normal \\
7 & Normal & Normal \\
\hline
\end{tabular}

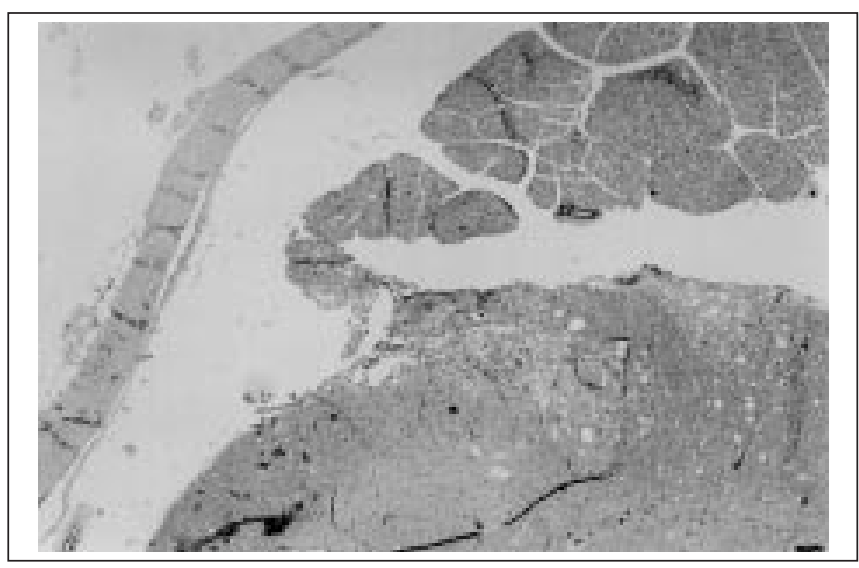

Figura 3 - Área Focal de Vacuolização na Superfície Dorso-Lateral da Medula e na Raiz Dorsal de Animal do Grupo 3. H E 40x

Quadro II - Observações Clínicas e Histológicas dos 7 Cães Pertencentes ao Grupo 3 que Receberam Ropivacaína a 1\%

\begin{tabular}{lll}
\hline Cão & Observações clínicas & Observações histológicas \\
\hline 1 & Normal & Normal \\
2 & Normal & Normal \\
3 & Normal & Normal \\
4 & Normal & Normal \\
5 & Normal & Normal \\
6 & Normal & Foco de necrose no tecido nervoso medular \\
7 & Normal & e raiz dorsal (menos que 5\%) \\
\hline
\end{tabular}

Não foram observadas alterações histológicas das meninges em nenhum animal, nos três grupos de estudo.

\section{DISCUSSÃO}

Esta pesquisa vem mostrar que a lidocaína a $2 \%$ determinou alterações histológicas medulares mais intensas que a ropivacaína a $1 \%$.

Quando grandes volumes de anestésico local são inadvertidamente injetados no espaço subaracnóideo, os efeitos de tamponamento e diluição do mesmo, que normalmente ocorrem com pequenos volumes, deixam de existir, possibilitando maior exposição da porção lombar e sacral da medula e das raízes nervosas sacrais ao anestésico local ${ }^{14}$.

É descrito que o anestésico local utilizado na anestesia peridural em concentrações que habitualmente não desencadeiam irritação no nervo são potencialmente capazes de produzir alterações neurológicas, se forem introduzidos em grandes volumes, no espaço subaracnóideo ${ }^{5}$.

Alguns autores constataram ${ }^{15}$ o aparecimento de alterações clínicas e histológicas após a administração de lidocaína a $1,5 \%$ em infusão contínua no espaço subaracnóideo de ratos. As alterações foram dependentes do tempo de exposição à droga e da dose administrada.

Em outra pesquisa, utilizando nervos ciáticos de rãs desprovidos de bainha nervosa, com a finalidade de estudar o potencial neurotóxico dos anestésicos locais, ficou demonstrado que a imersão do nervo por 5 minutos em uma solução de lidocaína hiperbárica a 5\% determinou bloqueio irreversível da condução do nervo. O bloqueio foi irreversível em $80 \%$ dos animais, quando o tempo de exposição foi de três minutos e a recuperação foi total com exposição de um minuto. Reduzindo-se a concentração da lidocaína para 1,5\% houve recuperação do potencial de ação em $70 \%$ dos nervos. Portanto, as alterações na condução da transmissão neural são também dependentes da concentração da droga ${ }^{16}$.

Também em nervos isolados de rãs, a lidocaína induziu perda irreversível da atividade dos impulsos dos nervos, de maneira dose-dependente, a partir de concentrações de 40 mmol, que correspondem à concentração de $1 \%$. A perda total da atividade neural aconteceu com concentrações de 80 mmol, ou seja, lidocaína a $2 \%$.

Em neurônios do gânglio da raiz dorsal de ratos, a lidocaína, em concentrações em torno de $30 \mathrm{mmol}$, em contato com o nervo durante quatro minutos, foi suficiente para induzir a morte dos neurônios ${ }^{17}$.

As concentrações de anestésico local que determinam morte celular são superiores àquelas que bloqueiam os canais de sódio de maneira reversível ${ }^{17}$.

Pouco se conhece sobre os mecanismos pelos quais os anestésicos locais produzem lesões nos nervos. Sabe-se apenas que, quando alterações clínicas se manifestam, há lesões suficientemente graves para produzir a perda da condução de algumas populações de fibras nervosas ${ }^{18}$.

Byers e col., $1973^{19}$, estudando os efeitos determinados pela lidocaína sobre os nervos de coelhos, em modelo experimental in vitro, demonstraram que concentrações tão baixas quanto $0,6 \%$, em contato por 60 minutos, determinaram bloqueio do transporte axoplasmático rápido e da condução do nervo. 
O transporte axoplasmático rápido é necessário para manter a estrutura e função do neurônio ${ }^{20}$. É possível que o anestésico local, em elevadas concentrações, determine bloqueio irreversível no transporte axoplasmático rápido e este fato possa contribuir para o desenvolvimento de paresias e paralisias que se observam após a realização de bloqueios regionais ${ }^{21}$.

A incidência de lesões neurológicas desencadeadas pelo anestésico local, nas doses habitualmente utilizadas na clínica, é pequena, possivelmente porque as concentrações da droga em contato com a membrana neuronal raramente atinjam valores que desencadeiem lesões.

A diluição do anestésico local no líquor contribui para reduzir a concentração do fármaco na membrana do neurônio, após a administração subaracnóidea ${ }^{5}$.

Fatores que limitam a difusão e a diluição da droga no líquor podem aumentar a concentração do anestésico que entrará em contato com a membrana do neurônio, desencadeando neurotoxicidade.

A morte celular não é necessariamente obrigatória para que existam alterações funcionais, porque reações tóxicas em qualquer população de células ou de receptores podem influenciar a função neurológica ${ }^{22}$.

Em nervos isolados de rãs, em modelo in vitro, o potencial de repouso da célula permaneceu inalterado mesmo quando o bloqueio de condução do nervo esteve comprometido de forma irreversível. Constatou-se que o gradiente iônico e a permeabilidade de repouso da membrana permaneceram intactos, porém os mecanismos necessários para que ocorressem a despolarização e a propagação do potencial de ação estavam permanentemente inutilizados. Desse modo, o anestésico local tornou os nervos sem função, sem, entretanto, destruí-los ${ }^{16}$

Essa teoria poderia explicar o aparecimento de sintomas clínicos sem as correspondentes alterações histológicas, como observado em dois animais do grupo 2, que apresentaram relaxamento parcial do esfíncter anal (área de distribuição do metâmero $\mathrm{S}_{3}$ ), com os cortes histológicos normais. Manteve-se sem alterações clínicas. o animal do grupo 3 que recebeu a ropivacaína a $1 \%$ e apresentou área focal de necrose da superfície dorso-lateral da medula, em área inferior a $5 \%$, no corte correspondente a $2 \mathrm{~cm}$ acima do local da punção. A ropivacaína foi manufaturada para ser utilizada em bloqueios de plexo e no espaço peridural. Estudo indica que a ropivacaína a $0,5 \%$ apresenta baricidade de 0,9999 , sendo considerada hipobárica ${ }^{12}$, o que pode ter favorecido o deslocamento cranial do anestésico e o aparecimento da lesão.

Também não existem, na literatura, até o momento, referências quanto aos efeitos que grandes volumes de ropivacaína poderiam determinar sobre o tecido nervoso e as meninges. Entretanto, sempre que há possibilidade de uma droga ser administrada no espaço subaracnóideo, mesmo de maneira acidental, é importante que se conheçam seus efeitos sobre o tecido nervoso e sobre o fluxo sangüíneo medular, visto que reduções importantes no fluxo sangüíneo podem determinar lesões neurológicas ${ }^{23}$.

Está descrito que a ropivacaína produziu significativa vasoconstrição nas pequenas e grandes arteríolas e nas vênulas da pia-máter de cães ${ }^{24}$.

Foram estudados os efeitos que a ropivacaína, nas concentrações de $0,5 \%$ e $2 \%$, exerce sobre o fluxo sangüíneo medular espinhal (FSME) de ratos, após administração por via subaracnóidea, durante período de 90 minutos. Os animais que receberam a ropivacaína a $2 \%$ apresentaram diminuição do FSME de aproximadamente $55 \%$, permanecendo estes valores em torno de cinco minutos e retornando ao normal gradualmente entre 20 e 40 minutos ${ }^{23}$.

Sabe-se que, após a administração subaracnóidea de anestésico local, a taxa metabólica da medula espinhal pode diminuir em decorrência do intenso bloqueio sensitivo e motor que é estabelecido ${ }^{25}$. Portanto, a ropivacaína pode ter reduzido o FSME, porque a taxa metabólica do tecido medular também diminuiu.

Contudo, a diminuição no FSME não parece ser a causa desencadeante das lesões neurológicas neste experimento, uma vez que, nos animais que receberam lidocaína a $2 \%$, as lesões foram mais intensas que quando se utilizou a ropivacaína a 1\%. Em trabalho pregresso ${ }^{26}$, a lidocaína hiperbárica a $5 \%$, portanto concentração maior do que a administrada neste estudo, não determinou isquemia do tecido nervoso de ratos.

Na presente pesquisa, nos três animais que apresentaram lesões histológicas do tecido nervoso da medula, estas se encontravam na superfície, onde há contato direto do anestésico local com o líquor. As lesões foram predominantemente na região dorsal da medula, estendendo-se até a substância branca, com exceção das de um animal do grupo 2, que foram encontradas em toda a superfície da medula. No animal que recebeu a ropivacaína, houve comprometimento da raiz posterior.

Em estudo sobre os efeitos neurotóxicos desencadeados pela tetracaína, após administração subaracnóidea, ficou demonstrado que as lesões se desenvolviam nas raízes dorsais e substância branca posterior da medula. Em local próximo à raiz posterior, imediatamente antes da sua entrada na substância branca medular, encontra-se uma zona desmielinizada, que contém neurônios desnudos. Estes neurônios são mais sensíveis à neurotoxicidade de drogas introduzidas no líquor ${ }^{27}$.

Para concluir, pode-se dizer que grandes volumes de lidocaína a $2 \%$ determinaram alterações histológicas sobre o tecido nervoso da medula espinhal, mais intensas que aquelas determinadas pela ropivacaína a 1\%. Ambas as drogas não ocasionaram lesões nas meninges de cães. 


\section{Effects of Spinal Administration of Large Volumes of $2 \%$ Lidocaine and $1 \%$ Ropivacaine on Spinal Cord and Meninges. Experimental Study in Dogs}

Eliana Marisa Ganem TSA, M.D., Pedro Thadeu Galvão Vianna TSA, M.D., Mariângela Marques, M.D., Yara Marcondes Machado Castiglia, TSA, M.D., Luiz Antonio Vane, TSA, M.D.

\section{INTRODUCTION}

Local anesthetics reversibly block cell membrane sodium channels thus preventing excitable tissue impulse conduction ${ }^{1}$. Lidocaine, an aminoamide anesthetic agent, is widely used due to its fast onset, potency and moderate duration ${ }^{2}$. Synthesized in 1948 by Löfgren, it was first spinally used in the concentration of $2 \%{ }^{3}$. In the mid 1950 s, it was introduced in the concentration of $5 \%$ and started to be used without enough research to evaluate the risk for potential neurological injuries triggered by its spinal administration. A multicentric study was published in 1969 involving 10,440 patients mostly from the obstetric clinic, in whom no severe neurological injury was observed ${ }^{4}$.

However, in the 1990s several cauda equina cases were described, which were associated to $5 \%$ hyperbaric lidocaine spinally administered through microcatheters ${ }^{5,6}$, small gauge pencil point needles ${ }^{7}$, after repeated injections due to inadequate block ${ }^{7,8}$ and, last but not least, after the injection of large $2 \%$ lidocaine volumes which should initially be administered in the epidural space but were accidentally introduced in the spinal space ${ }^{9-11}$.

Cauda equina syndrome is characterized by urinary and intestinal dysfunction, loss of perineal sensitivity and variable degrees of lower limb muscle weakness.

Nothing is known about the ability of ropivacaine, an amidoamine anesthetic agent prepared as pure $\mathrm{S}$ enantiomer, to trigger neurotoxicity ${ }^{12}$.

This study aimed at evaluating the effects of large spinal $2 \%$ lidocaine and $1 \%$ ropivacaine volumes on the histology of spinal cord nervous tissue and meninges of dogs.

\section{METHODS}

After the Animal Experiment Ethics Committee approval, 21 adult dogs of both genders and undefined breed, weighing 5 to $14 \mathrm{~kg}$ and with spinal length of 50 to $66 \mathrm{~cm}$ were used. Dogs were randomly distributed in 3 groups of 7 animals differentiated by the spinally administered solution: $\mathrm{G} 1$ - saline solution; G2 - 2\% lidocaine; G3 - 1\% ropivacaine.

The same experimental sequence was adopted for all animals. After a 12-hour fast with free access to water, dogs received $30 \mathrm{mg}^{\mathrm{kg}}{ }^{-1}$ intravenous sodium pentobarbital, were intubated and maintained in spontaneous ventilation in a circle system with $100 \%$ oxygen inspired fraction. Right femoral vein was dissected and catheterized for hydration with lac- tated Ringer's solution and right femoral artery was dissected and catheterized for mean blood pressure measurement.

Pulse oximetry was installed to measure oxygen peripheral saturation. Sensor was placed on dogs' tongue and a connector was installed close to the intubation cannula to measure carbon dioxide expired fraction $\left(\mathrm{CO}_{2}\right)$.

Animals were placed in the prone position on a Claude Bernard's trough and the distance between occipital protuberance and lumbosacral space was measured to obtain spinal length. This length was used to calculate the solution volume to be administered in the spinal space.

Skin and hair were cleaned with soap and water in an area of approximately $10 \mathrm{~cm}$ around $L_{6}-L_{7}$ interspace, followed by washing with water, disinfection with iodine alcohol and placement of sterilized drapes.

The two tuberosities of the iliac bone and of the spinal process of the last lumbar vertebra were palpated. The lumbosacral space was located immediately below. Sliding the second finger in the cephalad direction, the next intervertebral space was $L_{6}-L_{7}$.

To perform spinal puncture, a 22G disposable Quincke needle was inserted through median access with an angle of approximately $45^{\circ}$ and the bevel in the cephalad direction. After crossing the aracnoid membrane, needle mandrel was removed and CSF leakage was obtained.

The previously selected solution was then slowly injected in one minute, in a volume corresponding to $1 \mathrm{ml}$ for every $10 \mathrm{~cm}$ distance between occipital protuberance and lumbosacral space $^{13}$.

After local anesthetic injection, animals were placed in the supine position and monitoring consisted of heart rate, mean blood pressure and respiratory movements amplitude and frequency. These parameters have helped evaluating anesthetic block extent.

Animals with arterial hypotension (mean blood pressure below $30 \%$ of baseline values) would receive additional lactated Ringer's $\left(20 \mathrm{ml}^{\mathrm{kg}}{ }^{-1}\right)$. Those with a mean blood pressure decrease equal to or higher than $50 \%$ received $0.5 \mathrm{mg} \cdot \mathrm{kg}^{-1}$ ephedrine.

Dogs with bradycardia (heart rate $\leq 50 \%$ of baseline values or $\leq 60$ beats per minute) received $20 \mu \mathrm{g} \cdot \mathrm{kg}^{-1}$ atropine.

Animals with respiratory arrest were treated with mechanically controlled ventilation until effective spontaneous ventilation recovery.

The level of anal sphincter relaxation and painful sensitivity in different dermatomes, such as hind and front interdigital membrane, tail and different dermatomes of the cervical, thoracic, lumbar and sacral regions were also observed during spinal block with the aid of a mouse-tooth forceps.

After spinal and intravenous anesthesia recovery, animals remained in clinical observation for 72 hours. The following were evaluated: anal sphincter relaxation, loss of hind paws muscle strength, ability to move the tail and changes in painful sensitivity. 
Animals were sacrificed by electrocution after previous anesthesia and spinal cord lumbar and sacral portions were removed in less than 3 minutes, being fixed in 10\% formalin.

Nervous tissue and meninges were ressected, starting approximately $5 \mathrm{~cm}$ above spinal puncture site and going to the end of the cauda equina in $1 \mathrm{~cm}$ intervals.

Sections were dyed by the hematoxilin-eosin method and evaluated under light microscopy with the pathologist blind to sample's experimental group.

Analysis of variance was used to evaluate homogeneity of groups in terms of weight and spinal length being considered significant $p<0.05$.

\section{RESULTS}

There were no significant differences among groups in weight and spinal length (Table I).

Table I - Weight $(\mathrm{kg})$ and Spinal Length $(\mathrm{cm})$ of Animals (Mean \pm SD)

\begin{tabular}{lccc}
\hline & $\begin{array}{c}\text { Saline Solution } \\
\text { (G1) }\end{array}$ & $\begin{array}{c}2 \% \text { Lidocaine } \\
\text { (G2) }\end{array}$ & $\begin{array}{c}\text { 1\% Ropivacaine } \\
\text { (G3) }\end{array}$ \\
\hline Weight $(\mathrm{kg})$ & $9.6 \pm 2.8$ & $9.1 \pm 1.9$ & $9.3 \pm 2.9$ \\
Spinal length $(\mathrm{cm})$ & $59 \pm 6$ & $62 \pm 5$ & $60 \pm 6$ \\
\hline
\end{tabular}

All G2 and G3 animals needed mechanical ventilation after spinal block due to respiratory muscle palsy and consequent respiratory arrest. They also presented arterial hypotension. In 11 animals, pressure decrease varied from $20 \%$ to $50 \%$, was transient and was corrected with additional intravenous hydration. The other 3 needed ephedrine.

All G2 and G3 animals had a heart rate decrease varying from $40 \%$ to $75 \%$ of baseline values. However, only 4 dogs needed atropine to restore heart rate.

No G1 animal had spinal cord clinical or histological changes (Figure 1).

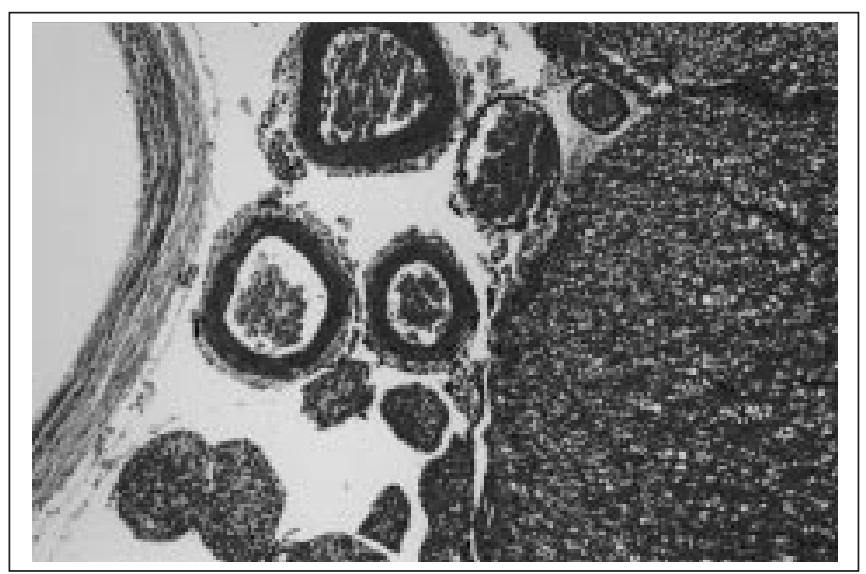

Figure 1 - Nervous Tissue, Blood Vessels and Normal Meninges of G1 Animals. HE 100x

Revista Brasileira de Anestesiologia

Vol. 53, № 3, Maio - Junho, 2003
There were histological changes in two G2 animals and clinical changes in one of them and in other two dogs of this group which, in turn, had no histological changes. One animal presenting histological changes had a band of necrosis on the spinal cord surface and on fasciculus gracillis, characterized by vacuolization with substance loss in $75 \%$ of the histological field, $2 \mathrm{~cm}$ above puncture site (Figure 2). Clinical evaluation has shown hind paws palsy and anal sphincter relaxation. A focus of medullary cone necrosis was found in the second animal, which occupied $30 \%$ of the histological field and had no clinical changes.

In two animals remaining with the anal sphincter partially relaxed after blockade, no histological changes were observed in spinal nervous tissue and meninges (Chart I).

Chart I - Clinical and Histological Observations of 7 G2 Dogs Receiving $2 \%$ Lidocaine

\begin{tabular}{lll}
\hline Dog & Clinical Observations & Histological Observations \\
\hline 1 & $\begin{array}{l}\text { Hind paws palsy and total anal } \\
\text { sphincter relaxation }\end{array}$ & $\begin{array}{l}\text { Strip necrosis reaching the } \\
\text { whole spinal cord surface and } \\
\text { fasciculus gracilis }(75 \%)\end{array}$ \\
2 & Partial anal sphincter relaxation & Normal \\
3 & Partial anal sphincter relaxation & Normal \\
4 & Normal & Normal \\
5 & Normal & $\begin{array}{l}\text { Focus of necrosis in the } \\
\text { medullary cone }(30 \%)\end{array}$ \\
6 & Normal & Normal \\
7 & Normal & Normal \\
\hline
\end{tabular}

One G3 animal presented with an area of vacuolization in the dorso-lateral surface of the spinal cord and in the dorsal root, occupying $5 \%$ of the histological field, approximately $2 \mathrm{~cm}$ above puncture site (Figure 3). All G3 animals remained clinically normal (Chart II).

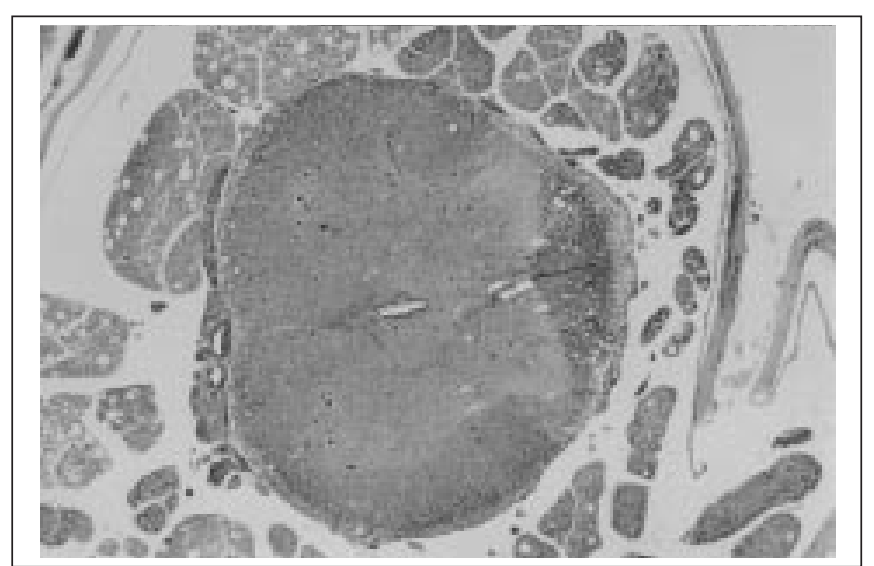

Figure 2 - Band Necrosis throughout Spinal Cord Surface and Gracilis Fascicle of G2 Animal. H E 20x 


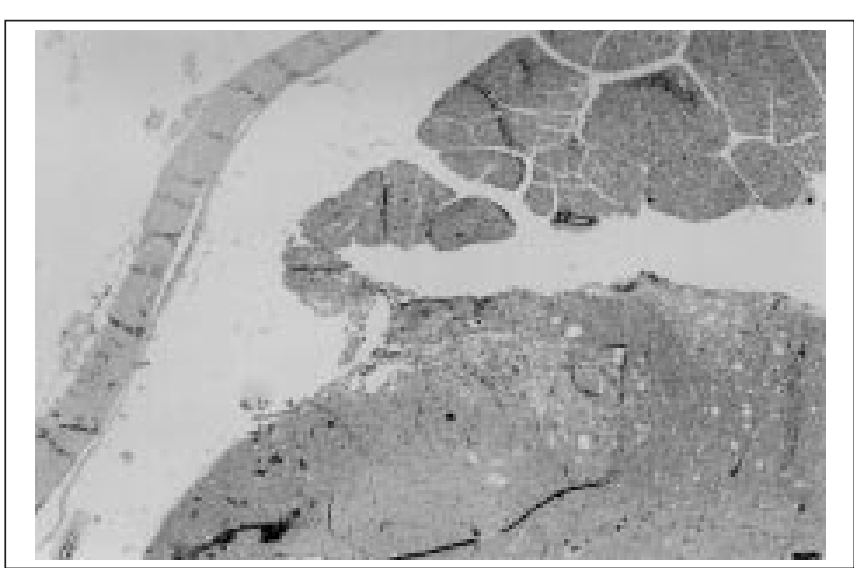

Figure 3 - Focal Vacuolization Area in Spinal Cord Dorso-Lateral Surface and Dorsal Root of G3 Animal. H E 40x

Chart II - Clinical and Histological Observations of 7 G3 Dogs Receiving 1\% Ropivacaine

\begin{tabular}{lll}
\hline Dog & Clinical Observations & Histological Observations \\
\hline 1 & Normal & Normal \\
2 & Normal & Normal \\
3 & Normal & Normal \\
4 & Normal & Normal \\
5 & Normal & Normal \\
6 & Normal & Focus of necrosis in spinal cord nervous \\
7 & Normal & tissue and dorsal root (less than 5\%) \\
\hline
\end{tabular}

There were no histological meningeal changes in all animals.

\section{DISCUSSION}

This research has shown that $2 \%$ lidocaine has determined more intense spinal cord histological changes as compared to $1 \%$ ropivacaine.

When large local anesthetic volumes are accidentally injected in the spinal space, anesthetic buffering and dilutional effects, which normally occur with low volumes, are no longer there, allowing a greater exposure of spinal cord lumbar and sacral portions and of sacral nervous roots to local anesthetics ${ }^{14}$.

It has been described that local anesthetics used in epidural anesthesia in concentrations normally not triggering nerve irritation, are potentially able to produce neurological changes if introduced in large volumes in the spinal space ${ }^{5}$. Some authors have observed ${ }^{15}$ clinical and histological changes after the administration of $1.5 \%$ lidocaine continuous infusion in the spinal space of mice. Changes were a function of the exposure time to the drug and of the dose.
In a different research, using sciatic nerves of frogs without nervous sheath and aiming at studying local anesthetic neurotoxic potential, it has been shown that the immersion of the nerve for 5 minutes in a solution of $5 \%$ hyperbaric lidocaine has determined an irreversible blockade of nerve conduction. Blockade was irreversible in $80 \%$ of animals when exposure time was 3 minutes and total recovery was obtained with 1-minute exposure. Decreasing lidocaine's concentration to $1.5 \%$, there has been action potential recovery in $70 \%$ of nerves. So, changes in neural transmission conduction are also dependent on drug concentration ${ }^{16}$.

In isolated nerves of frogs, lidocaine has also induced irreversible loss of nerve impulse activity in a dose-dependent manner, as from $40 \mathrm{mmol}$ concentrations, which correspond to $1 \%$. Total neural activity loss was seen with $80 \mathrm{mmol}$, that is $2 \%$ lidocaine.

In dorsal root ganglia neurons of mice, lidocaine in concentrations of approximately $30 \mathrm{mmol}$ in contact with the nerve for 4 minutes was enough to induce neuronal death ${ }^{17}$.

Local anesthetic concentrations determining cell death are higher than those blocking sodium channels in a reversible manner ${ }^{17}$.

Little is known about the mechanisms through which local anesthetics induce nervous injuries. What is known is that when there are clinical changes, there are injuries severe enough to produce conduction loss in some nervous fiber populations ${ }^{18}$

Byers et al., ${ }^{19}$ in 1973, studying lidocaine effects on rabbit nerves in an experimental in vitro model, have shown that concentrations as low as $0.6 \%$ in a 60 -minute contact would block fast axoplasma transport and nervous conduction.

Fast axoplasma transport is needed to maintain neuronal structure and function ${ }^{20}$. It is possible that high local anesthetic concentrations would determine irreversible block of the fast axoplasma transport and this could contribute to paresthesias and paralysis seen after regional blockade ${ }^{21}$. The incidence of neurological injuries triggered by local anesthetics, in doses currently used in the clinical practice, is low probably because drug concentration in contact with neuronal membrane seldom reaches injury-triggering values.

Local anesthetic dilution in CSF contributes to decrease drug concentration in the neuronal membrane after spinal administration ${ }^{5}$.

Factors limiting drug diffusion and dilution in CSF may increase anesthetic concentration in touch with the neuronal membrane, thus triggering neurotoxicity.

Cell death is not necessarily mandatory for the existence of functional changes because toxic reactions in any cell or receptor population may influence neurological function ${ }^{22}$. In isolated nerves of frogs in an in vitro model, cell rest potential remained unchanged even when nervous conduction blockade was irreversibly impaired. It was observed that membrane's ionic gradient and rest patency remained intact, but mechanisms needed for depolarization and action potential propagation were permanently impaired. This way, local anesthetics made nerves functionless, without however destroying them ${ }^{16}$. 
This theory could explain clinical symptoms without corresponding histological changes, as seen in two G2 animals which presented partial anal sphincter relaxation (distribution area of metamer $S_{3}$ ), with normal histological findings. There were no clinical changes in the $\mathrm{G} 3$ animal which received $1 \%$ ropivacaine and presented less than $5 \%$ focal necrosis in spinal cord dorso-lateral region, in the section corresponding to $2 \mathrm{~cm}$ above puncture site.

Ropivacaine was developed to be used in plexus and epidural space blocks. Astudy has shown that $0.5 \%$ ropivacaine has a baricity of 0.9999 being considered hypobaric ${ }^{12}$, fact which may have favored anesthetic cranial shift and the appearance of the lesion.

To date, there are also no data in the literature on the effects of high ropivacaine volumes on nervous tissue and meninges. However, whenever there is the possibility of a drug being administered in the spinal space, even accidentally, it is important to understand its effects on nervous tissue and on medullary blood flow, since a major blood flow decrease may determine neurological deficits ${ }^{23}$.

It has been described that ropivacaine produces major vasoconstriction in small and large arterioles and in pial venules of dogs ${ }^{24}$.

The effects of $0.5 \%$ and $2 \%$ ropivacaine on spinal cord blood flow (SCBF) of rats after spinal administration were evaluated during a 90 -minute period. Animals receiving $2 \%$ ropivacaine showed a SCBF decrease of approximately $55 \%$ which remained for approximately 5 minutes and gradually returned to normal within 20 to 40 minutes ${ }^{23}$

It is known that after local anesthetic spinal administration, spinal cord metabolic rate may decrease as a consequence of intense sensory and motor block ${ }^{25}$. So, ropivacaine may have decreased SCBF because medullary tissue metabolic rate has also decreased.

However, SCBF decrease did not seem to be the triggering factor for neurological deficits in our experiment, since animals receiving $2 \%$ lidocaine had more severe injuries as compared to those receiving $1 \%$ ropivacaine. In a previous study ${ }^{26}, 5 \%$ hyperbaric lidocaine, thus a higher concentration than that used in our study, has not determined nervous tissue ischemia in mice.

In our study, in the three animals with spinal cord histological changes, these were superficial and developed where there was direct contact of CSF with the nervous tissue. Injuries were predominantly in spinal cord dorsal region, extending to the white matter, with the exception of $1 \mathrm{G} 2$ animal where they were found throughout the spinal cord surface. The animal receiving ropivacaine had posterior root involvement.

In a study on neurotoxic effects triggered by spinal tetracaine, it has been shown that injuries were developed in spinal cord dorsal roots and posterior white matter. In a site close to the posterior root, immediately before its insertion in the spinal cord white matter, there is a demyelinized region with nude neurons. These neurons are more sensitive to neurotoxicity of drugs introduced in the CSF ${ }^{27}$.

In conclusion, large $2 \%$ lidocaine volumes determine more severe histological changes on spinal cord nervous tissue as compared to $1 \%$ ropivacaine. Both drugs caused no meningeal injuries in dogs.

\section{REFERÊNCIAS - REFERENCES}

01. de Jong RH - Local Anesthetic Pharmacology, em: Brown DL Regional Anesthesia and Analgesia. Philadelphia: W. B. Saunders, 1996;8:124-142.

02. Veering BT - Local Anesthetics, em: Brown DL - Regional Anesthesia and Analgesia. Philadelphia: W. B. Saunders, 1996;12: 188-207.

03. Herman N - Neurologic complications of regional anesthesia. Semin Anesth Perioper Med Pain, 1998;17:64-72.

04. Phillips OC, Ebner H, Nelson AT et al - Neurologic complications following spinal anesthesia with lidocaine: a prospective review of 10.440 cases. Anesthesiology, 1969;30:284-289.

05. Rigler ML, Drasner K, Krejcie TC et al - Cauda equina syndrome after continuous spinal anesthesia. Anesth Analg, 1991;72: 275-281.

06. Lambert DH, Hurley RJ - Cauda equina syndrome and continuous spinal anesthesia. Anesth Analg, 1991;72:817-819.

07. Beardsley D, Holman S, Gantt R et al - Transient neurological deficit after spinal anesthesia: local anesthetic maldistribution with pencil point needles? Anesth Analg, 1995;81:314-320.

08. Loo CC, Irestedt L - Cauda equina syndrome after spinal anaesthesia with hyperbaric $5 \%$ lignocaine: a review of six cases of cauda equina syndrome reported to the Swedish pharmaceutical insurance 1993-1997. Acta Anaesthesiol Scand, 1999;43: 371-379.

09. Drasner K, Rigler M, Sessler DI et al - Cauda equina syndrome following intended epidural anesthesia. Anesthesiology, 1992;77:582-585.

10. Cheng ACK - Intended epidural anesthesia as possible cause of cauda equina syndrome. Anesth Analg, 1994;78:157-159.

11. Lee DS, Bui T, Ferrarese $\mathrm{J}$ et al - Cauda equina syndrome after incidental total spinal anesthesia with $2 \%$ lidocaine. J Clin Anesth, 1998;10:66-69.

12. McClure JH - Ropivacaine. Br J Anaesth, 1996;76:300-307.

13. Hendrix PK, Raffe MR, Robinson EP et al - Epidural administration of bupivacaine, morphine, or their combination for postoperative analgesia in dogs. J Am Vet Med Assoc, 1996;209: 598-607.

14. Kane RE - Neurologic deficits following epidural or spinal anesthesia. Anesth Analg, 1981;60:150-161.

15. Li DF, Bahar M, Cole F et al - Neurological toxicity of the subarachnoid infusion of bupivacaine, lignocaine or 2-chloroprocaine in the rat. Br J Anaesth, 1985;57:424-429.

16. Lambert LA, Lambert DH, Strichartz GR - Irreversible conduction block in isolated nerve by high concentrations of local anesthetics. Anesthesiology, 1994;80:1082-1093.

17. Gold MS, Reichling DB, Hampl KF et al - Lidocaine toxicity in primary afferent neurons from the rat. J Pharmacol Exp Ther, 1998;285:413-421.

18. Kalichman MW - Physiologic mechanisms by which local anesthetics may cause injury to nerve and spinal cord. Reg Anesth, 1993;18:448-452.

19. Byers MR, Fink BR, Kennedy RD et al - Effects of lidocaine on axonal morphology, microtubules, and rapid transport in rabbit vagus nerve in vitro. J Neurobiol; 1973;4:125-143.

20. Malinovsky JM, Pinaud M - Neurotoxicité des agents administrés par voie intrathécale. Ann Fr Anesth Réanim, 1996;15:647-658. 
21. Dahlström A - "Axoplasmatic Transport"- The catering and communication system within nerve cells. Anesthesiology, 1974;41:537-541.

22. Myers RR, Sommer C - Methodology for spinal neurotoxicity studies. Reg Anesth, 1993;18:439-447.

23. Kristensen JD, Karlsten R, Gordh T - Spinal cord blood flow after intrathecal injection of ropivacaine: a screening for neurotoxic effects. Anesth Analg, 1996;82:636-640.

24. lida $\mathrm{H}$, Watanabe $\mathrm{Y}$, Dohi $\mathrm{S}$ et al - Direct effects of ropivacaine on spinal pial vessels in canine - comparison with bupivacaine. Anesthesiology, 1995;83:A828.

25. Crosby G - Local spinal cord blood flow and glucose utilization during spinal anesthesia with bupivacaine in conscious rats. Anesthesiology, 1985;63:55-60.

26. Benveniste $H$, Qui $H$, Hedlund $L W$ et al - In vivo diffusion-weighted magnetic resonance microscopy of rat spinal cord: effect of ischemia and intrathecal hyperbaric 5\% lidocaine. Reg Anesth Pain Med, 1999;24:311-318.

27. Takenami T, Yagishita S, Asato $F$ et al - Neurotoxicity of intrathecally administered tetracaine commences at the posterior roots near entry into the spinal cord. Reg Anesth Pain Med, 2000;25:372-379.

\section{RESUMEN}

Ganem EM, Vianna PTG, Marques M, Castiglia YMM, Vane LA Efectos de la Administración Subaracnóidea de Grandes Volúmenes de Lidocaína a 2\% y Ropivacaína a 1\% sobre la Médula Espinal y las Meninges. Estudio Experimental en Perros

JUSTIFICATIVA Y OBJETIVOS: La inyección de grandes volúmenes de anestésico local en el espacio subaracnóideo, después de punción dural accidental, es complicación de la anestesia peridural. El objetivo de esta pesquisa fue investigar las posibles alteraciones clínicas e histológicas desencadenadas por grandes volúmenes de lidocaína a $2 \%$ y ropivacaína a 1\%, simulando una inyección subaracnóidea accidental, en perros.

MÉTODO: Veintiún perros fueron distribuidos aleatoriamente en 3 grupos, que recibieron por vía subaracnóidea: G1 - cloreto de sodio a 0,9\%; G2 - lidocaína a $2 \%$ y G3 - ropivacaína a $1 \%$. La punción subaracnóidea fue realizada en el espacio intervertebral $L_{6}-L_{7}$. El volumen de anestésico local administrado fue de $1 \mathrm{ml}$ para cada $10 \mathrm{~cm}$ de distancia entre la protuberancia occipital y el espacio lomosacral (5 - 6,6 ml). Después de 72 horas de observación clínica los animales fueron sacrificados y fue removida la porción lomosacral de la médula para examen histológico, por microscopia óptica.

RESULTADOS: Ningún animal del G1 presentó alteraciones clínicas o histológicas de la médula espinal. Fueron observados dos casos de necrosis del tejido nervioso en G2, más cambios clínicos, en solamente uno de estos perros y en otros dos animales que no presentaron alteraciones histológicas. Fue encontrada necrosis focal del tejido nervioso medular en un animal de G3. Todos los animales de G3 permanecieron clínicamente normales.

CONCLUSIONES: Se concluye que grandes volúmenes de lidocaína a $2 \%$ determinaron alteraciones clínicas e histológicas más intensas que los de ropivacaína a $1 \%$. 